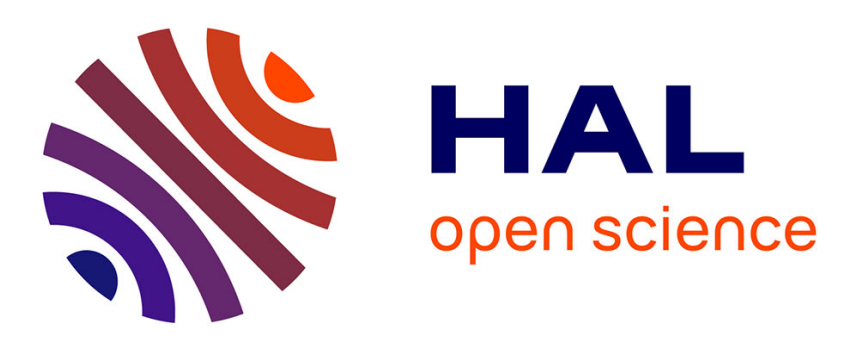

\title{
In-situ measurement of non-steady flows using optical feedback interferometry
}

\author{
Evelio Esteban Ramírez-Miquet, Antonio Luna Arriaga, Adam Quotb, O. \\ Sotolongo-Costa, Julien Perchoux
}

\section{- To cite this version:}

Evelio Esteban Ramírez-Miquet, Antonio Luna Arriaga, Adam Quotb, O. Sotolongo-Costa, Julien Perchoux. In-situ measurement of non-steady flows using optical feedback interferometry. 2015 IEEE International Conference on Industrial Technology, Mar 2015, Séville, Spain. pp.1469-1473, 10.1109/ICIT.2015.7125304 . hal-01167816

\section{HAL Id: hal-01167816 https://hal.science/hal-01167816}

Submitted on 24 Jun 2015

HAL is a multi-disciplinary open access archive for the deposit and dissemination of scientific research documents, whether they are published or not. The documents may come from teaching and research institutions in France or abroad, or from public or private research centers.
L'archive ouverte pluridisciplinaire HAL, est destinée au dépôt et à la diffusion de documents scientifiques de niveau recherche, publiés ou non, émanant des établissements d'enseignement et de recherche français ou étrangers, des laboratoires publics ou privés. 


\title{
In-situ Measurement of Non-steady Flows Using Optical Feedback Interferometry
}

\author{
E.E. Ramírez-Miquet, ${ }^{* \dagger \dagger}$ A. Luna Arriaga, ${ }^{* \dagger} \dagger$ A. Quotb,${ }^{* \dagger}$ O. Sotolongo-Costa,,${ }^{\S}$ and J. Perchoux ${ }^{* \dagger}$ \\ ${ }^{*}$ CNRS, LAAS, 7 avenue du colonel Roche, F-31400 Toulouse, France \\ †Univ de Toulouse, INP, LAAS, F-31400 Toulouse, France \\ $\ddagger$ Centro de Aplicaciones Tecnológicas y Desarrollo Nuclear (CEADEN), Calle 30, No. 502, Miramar 11300, La Habana, Cuba \\ ${ }^{\S}$ Cátedra de Sistemas Complejos "Henri Poincaré", Universidad de La Habana, Vedado 10400, La Habana, Cuba \\ ๑Departamento de Física, Universidad Autónoma del Estado de Morelos, Cuernavaca 62209 Morelos, México \\ " alunaarr@laas.fr
}

\begin{abstract}
We present a real-time system for flow measurement in millifluidic configuration based on optical feedback interferometry. The sensing method exploits the power spectral density information of the acquired signals affected by the backscattered light of particles contained in a fluid. We validate experimentally the method on-site by reconstructing the periodicity of nonsteady flows of a peristaltic pump. This demonstrates potential applications for flow assessments in closed liquid-filled circuits.
\end{abstract}

\section{INTRODUCTION}

Optical feedback interferometry (OFI) is a well-established non-destructive technique for sensing physical parameters [1]. Mainly used for velocity [2], absolute distance [3], vibration and displacement of solid targets [4]-[6], this technique has been recently extended to flow measurements [7].

In the context of flow velocity measurement, the laser beam is pointed to a moving particle and part of the backscattered light is fed-back into the laser cavity. Due to Doppler effect, this feedback induces changes in the laser behavior affecting directly the optical emission power and wavelength. Since the laser itself is used as the emitter and photo-receiver, the technique employs minimal optical elements and requires relatively low-cost components [8].

Even though most optical sensors based on OFI have been primary developed for sensing purposes in mechatronics, they have demonstrated accurate and robust performance to characterize continuous and steady flows in milli and microchannels [9]-[11]. The implementation of optical feedback sensors in fluidics applications extends their utility as alternative measurement devices in some fields such as chemical and biomedical engineering [12], where bulky and expensive devices based on imaging are employed for the assessment of fluid flows and microcirculation [13].

So far, to the best of our knowledge, often reported optical feedback flowmeters have been tested for measuring steady and unidirectional flows [9], [10], [12]. Moreover, previously reported signal analysis usually require careful and time consuming calculation implying post-processing for flow characterization, thus constraining a real-time implementation of this technology.

Norgia et al. [14] proposed an analog low-cost sensor for real-time blood flow measurement. Their system validation was done using a peristaltic pump with three pressing members ensuring continuous pumping of fluids. However, their algorithm based on a probability density function required specific infield estimation for calibration. It also needed a compounded filter bank and application-specific circuit design for signal conditioning before a real-time implementation in LabVIEW ${ }^{\circledR}$ software.

In this paper, we present an OFI flowmeter in a millifluidic configuration for real-time assessment of non-steady flows, i.e. when the fluid is not being pressed momentarily by the pump squeezers. We propose an in-situ calculation of a quantitative parameter allowing to easily evaluate the periodicity of non-steady flows without associated post-processing and with straightforward calibration. This approach is targeted to interrogate closed liquid-filled circuits during operation and it is also directly linked to the possibility of characterizing flows even if the technical features of the pumping device are unknown.

\section{OFI TECHNIQUE FOR FLOW MEASUREMENTS}

Flow measurements using OFI technique require a minimal concentration of scattering particles embedded in the fluid. Therefore, it is understood that these tracer particles follow the hydrodynamic properties of the fluid to guarantee an accurate local velocity measure of the flow. This condition allows the successful implementation of OFI-based optofluidic systems for velocity profiling in micro-channels [9] and particle tracking with size estimation [15].

The more generalized sensing methods using OFI in laser diodes are the measurement of the optical power changes in the photodiode (PD) included in the laser's module or by directly measuring the voltage changes in the junction terminals due to optical feedback [16]. In any case, the relation of the emission power $P_{t}$ with target velocity is given by the formula:

$$
P_{t}=P_{0}\left\{1+\cos \left[2 \pi \frac{2\left(L+v_{t} t\right)}{c}\right]\right\}
$$

where $P_{0}$ is the optical power of the laser without feedback, $L$ is the distance between the target and the laser, $c$ is the speed of light, $t$ is time and $v_{t}$ is the projection of the target velocity onto the laser beam propagation axis. 
OFI signals from fluid flows contain information on the velocity of its embedded particles in a wide range. The analysis of power spectral density using Fast Fourier Transform (FFT) of temporal signals allows the direct calculation of flow velocity, which is related to the fundamental Doppler frequency $f_{\mathrm{D}}$ in the spectrum as follows:

$$
f_{\mathrm{D}}=\frac{2 n v_{\mathrm{t}}}{\lambda}
$$

where $n$ is the refractive index of the surrounding medium of the flow, $v_{t}$ is the projection of the fluid velocity onto the laser beam propagation axis and $\lambda$ is the laser wavelength.

Kinematic properties of the flow can be determined from Eq. (2), when a low concentration of particles in the fluid allows to recognize the fundamental Doppler frequency on the spectrum. When the number of particles in the flow increases considerably, multiple scattering effects arise, making difficult the identification of Doppler frequency shifts.

\section{PROPOSED METHOD}

It was demonstrated in [9], [14] that under low particles concentration (single scattering regime) there is a linear relation between flow rate and fundamental Doppler frequency. Since increasing the number of scatterers makes this protocol not suitable for fluids carrying high number of particles, we propose a calculation denoting the relative variation of the target flow.

This calculation consists on the integration of the amplitude differences in the spectrum (in $\mathrm{dB}$ ) of a set of OFI signals with and without flow. As a matter of fact, the power spectral properties of an acquired signal differ depending on the flow variations in time. Therefore, by subtracting the spectrum of two different measurements and integrating this difference, a variety of flow behaviors can be identified. This process is expressed by Eq. (3):

$$
\mathbf{M}_{\mathrm{f}}=\sum_{f_{\min }=0}^{f_{\max }=F_{\mathrm{s}} / 2}\left|\mathrm{OFI}_{\text {flow }}-\mathrm{OFI}_{\text {no flow }}\right|
$$

where $f$ is the frequency in the spectrum which ranges from 0 to half the sampling frequency $\left(F_{\mathrm{s}}\right)$ of the acquisition system, $\mathrm{OFI}_{\text {no flow }}$ is the obtained spectrum when the fluid does not move, $\mathrm{OFI}_{\text {flow }}$ is the spectrum when the fluid is moving and $\mathrm{M}_{\mathrm{f}}$ is a parameter related to the flow variations.

The quantitative value $\mathrm{M}_{\mathrm{f}}$ is thus calculated in a continuous manner by subtracting the above mentioned spectrums as represented in Fig. 1. The calculation of $\mathrm{OFI}_{\text {no flow }}$ consists on a calibration step accounting for the local inherent noise added to OFI signals during a measurement. In order to provide a reliable reference we use two averaging procedures. The first is made between a set of FFTs of predefined size calculated along the signal acquired at a given sampling rate. The second averaging consists on repeating the above procedure at three different times so as to discard isolated noise conditions. Once calculated the average, this spectrum is smoothed with a running average filter to further increase the repeatability of calibration step.
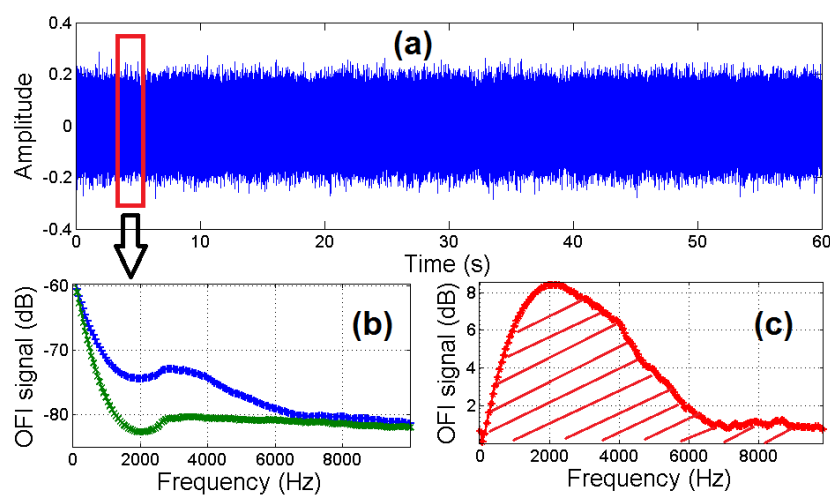

Fig. 1: Overview of proposed method performing over a continuously acquired time domain OFI signal (a). Spectrums of $\mathrm{OFI}_{\text {flow }}$ and $\mathrm{OFI}_{\text {no flow }}$ (b) and their difference (c).

The calculation of $\mathrm{OFI}_{\text {flow }}$ can be established as a single FFT along the signal acquired at a given sampling rate. According to the noise conditions in a setup, performing an averaging between a set of FFTs will reduce the influence of extraneous noise. However, this trade-off between calculation time and noise reduction needs to be considered while attempting on-site calculation. Since flow speed induces a Doppler shift that is contained in a close range of frequencies, it is convenient to truncate a representative bandwidth from the spectrum to reduce computation resources. Cut-off frequency limits can be determined by inspecting the power spectral densities acquired for a minimum and maximum flow rate of a particular application. (e.g. for the experiment described in following section, we selected $f_{\min }=100 \mathrm{~Hz}$ and $f_{\max }=$ $50 \mathrm{kHz}$ ).

\section{A. Real-time implementation}

The proposed method can be performed by a processing hardware as represented in Fig. 2. From the basic OFI configuration, an analog signal containing the information relative to the particle's flow speed is digitized with an analog-todigital converter (ADC) and fed within a circular buffer to the processing hardware. The FFT and truncation operation from Eq. (3) provide then a continuous $\mathrm{M}_{\mathrm{f}}$ calculation.

Referring back to the temporal OFI signal represented in Fig. $1, \mathrm{M}_{\mathrm{f}}$ is performed by block segments. From signal processing theory, the compromise between block size $(N)$ and acquisition sampling rate establishes the frequency bin resolution $\left(F_{\text {bin }}\right)$ as $F_{\text {bin }}=F_{\mathrm{s}} / N$, thus imposing a hard realtime constraint to be inferior to $1 / F_{\text {bin }}$.

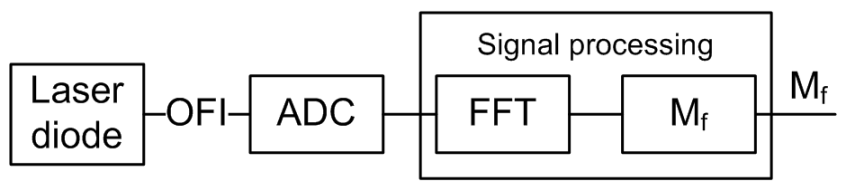

Fig. 2: Block diagram of the proposed real-time system's implementation. 


\section{EXPERIMENT}

The optical setup used during the experimental series is shown in Fig. 3. It consists on a laser diode (Thorlabs L785P090) emitting at $785 \mathrm{~nm}$ and driven by an injection current of $60 \mathrm{~mA}$. The laser is coupled to a single lens of $8 \mathrm{~mm}$ focal length and the collimated radiation is pointed to a transparent tube with an angle of $80^{\circ}$. With this configuration we found an optimal signal to noise ratio (SNR) in the spectrum. For the fluid, we tested a dilution of full cream milk concentrated by mass at $20.2 \%$ in water.

A two-squeezer peristaltic pump (Seko PR1) designed for warewashing in catering industry was used during the experiments. It includes an analog potentiometer with drawn marks to regulate the flow rate.

The pump flow rate has been previously characterized to obtain a countermeasurement for the experiment. During 1 min we measured the volume of fluid drained by the pump in a beaker for every position of the potentiometer. A USB camera is used to trail a single fluid bubble while one of the pressing members of the pump is squeezing the tube to force the flowing. A simple processing of a sequence of images allowed the determination of the distance $(S)$ traveled by the milk in the intermittent flow regime, which yielded $34.2 \mathrm{~mm}$ as shown in Fig. 4.

During OFI measurements, signals from the laser's photodiode containing 4096 samples were acquired at $500 \mathrm{kHz}$ with a National Instruments data acquisition card (USB-6361) connected to a personal computer.

Flow rate was estimated by using the typical equation for cylindrical tubes $Q=v A$, where $A$ is cross-section of the tube which measured radius is $r=1.75 \mathrm{~mm}$ and $v$ is the averaged velocity of the fluid given by $v=S / \tau$, being $\tau$ the periodicity determined from the reconstructed signal of the pump by plotting $\mathrm{M}_{\mathrm{f}} v$ s. time during 1 min using our proposed method in Eq. (3) for the whole range of the pump marks.

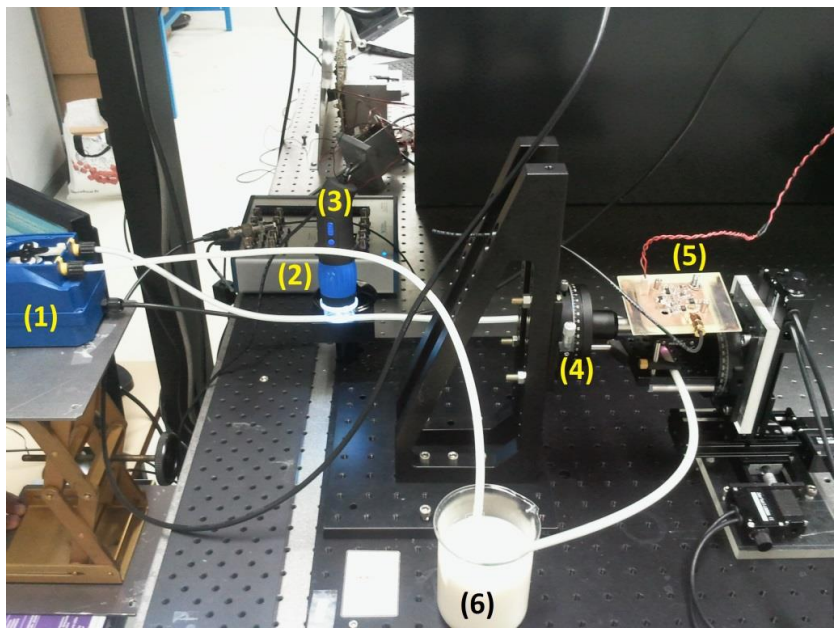

Fig. 3: Experimental setup: (1) peristaltic pump, (2) acquisition card, (3) camera, (4) goniometer, (5) laser and associated electronics, (6) fluid.
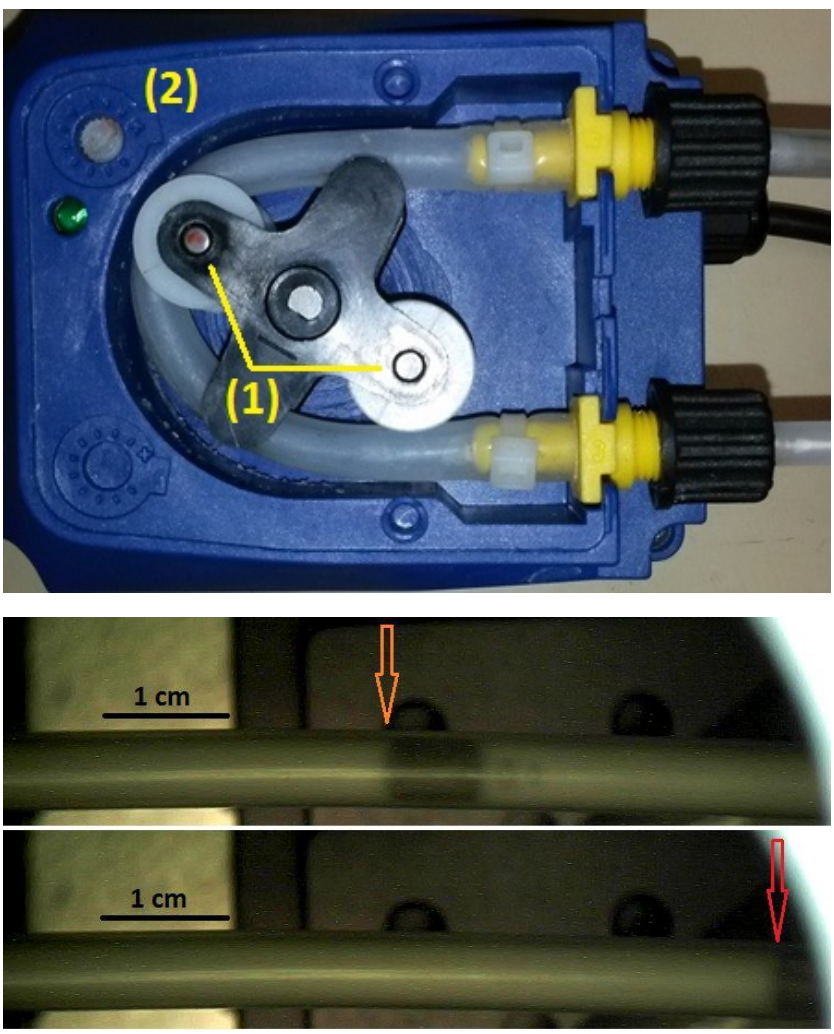

Fig. 4: Procedure to determine a countermeasurement for fluid's displacement. (1) Pump squeezers, (2) Potentiometer.

\section{RESULTS AND DISCUSSION}

As a first step, we assess the flows using post-processing in order to have a comparative reference. Then, we performed the real-time analysis.

The analysis of signals acquired during $1 \mathrm{~min}$ to reconstruct the periodicity of the pump is shown in Fig. 5. Four plots of $\mathrm{M}_{\mathrm{f}} v s$. time are represented, corresponding to four flow rates, from the minimum value in the potentiometer to higher values. It can be observed how the changes in pump rate lead to an increment of the periodicity denoted by the minimal values of $\mathrm{M}_{\mathrm{f}}$ corresponding to the instant when the liquid flow stops. Let us highlight the fact that this period-reconstruction algorithm allows to observe those instants where the nonsteady regime produces a rapid suction and the flow is pulled back, as confirmed by the spikes in the vicinity of the minimal values in the figure.

Using the reconstruction of the periodicity of the pump, we proceeded to validate the linear relation between flow rate and parameter $\mathbf{M}_{\mathrm{f}}$. These last values were averaged in the intervals where the pressing squeezers were forcing the fluid to flow. Fig. 6 shows the errorbars of flow rate $v s$. $\mathrm{M}_{\mathrm{f}}$ determined by the two methods for eight positions of the pump's potentiometer, depicting a good agreement of both measurements. A fitting of these experimental values shows a linear regression with slope equal to $0.004, \mathrm{M}_{\mathrm{f}}$-axis intercept equal to -7.76 and $R^{2}=0.99$, which is consistent with previous reports where this linear regression was also obtained [9], [14].

For the real-time validation, the algorithm has been im- 

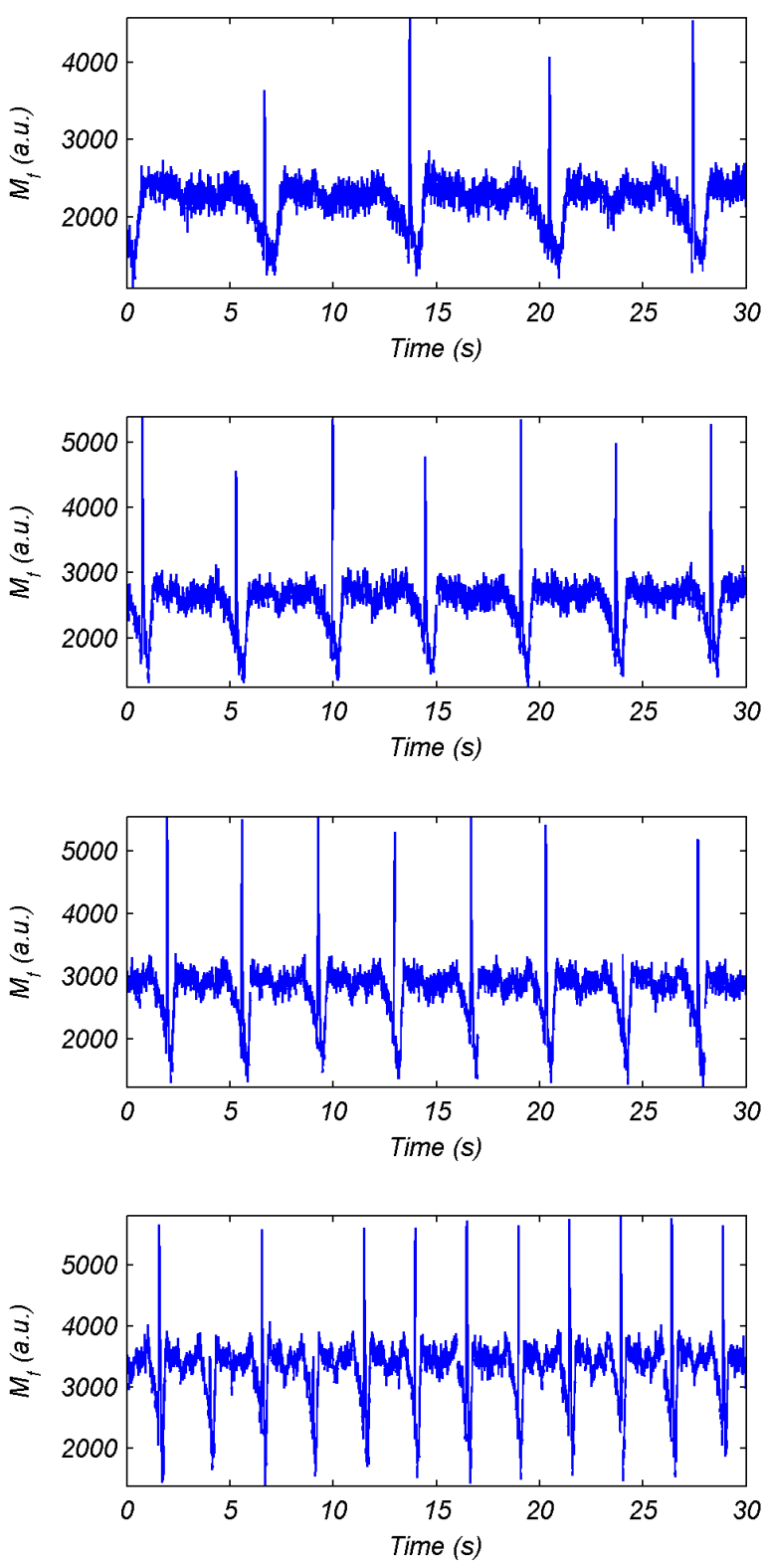

Fig. 5: Reconstructed pump period at different flow rates (showing $30 \mathrm{~s}$ for clarity), from top to bottom: pump's potentiometer in 1st, 4th, 6th and 8th position marks.

plemented in Matlab ${ }^{\circledR}$ software using the data acquisition toolbox. Since the host operative system cannot guarantee determinism to plot the calculated points, we have relaxed the real-time constraint $(1 / \mathrm{dt})$ by averaging an increased number of FFTs and thus increasing the acquisition time before a new arrival of samples. Thus, the experimental imposed constraint of $8.19 \mathrm{~ms}$ (for $F_{\mathrm{s}}=500 \mathrm{kHz}$ and $N=4096$ points) has been extended to $81.92 \mathrm{~ms}$ by averaging 10 FFTs for each point. As can be appreciated in the developed front panel of the instrument (Fig. 7), the pump's period reconstruction agrees with the off-line characterization. The added value of this approach is the possibility to assess and control fluidics applications.

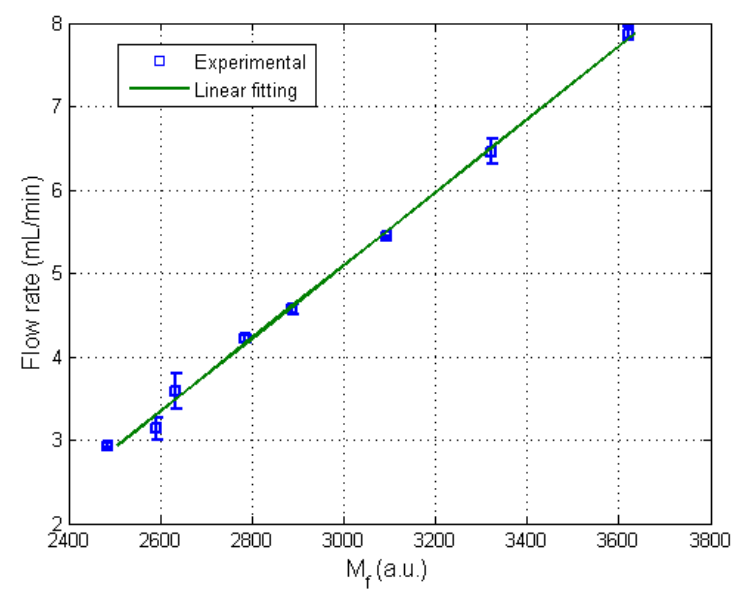

Fig. 6: Linear relationship of Flow rate vs. $\mathrm{M}_{\mathrm{f}}$.

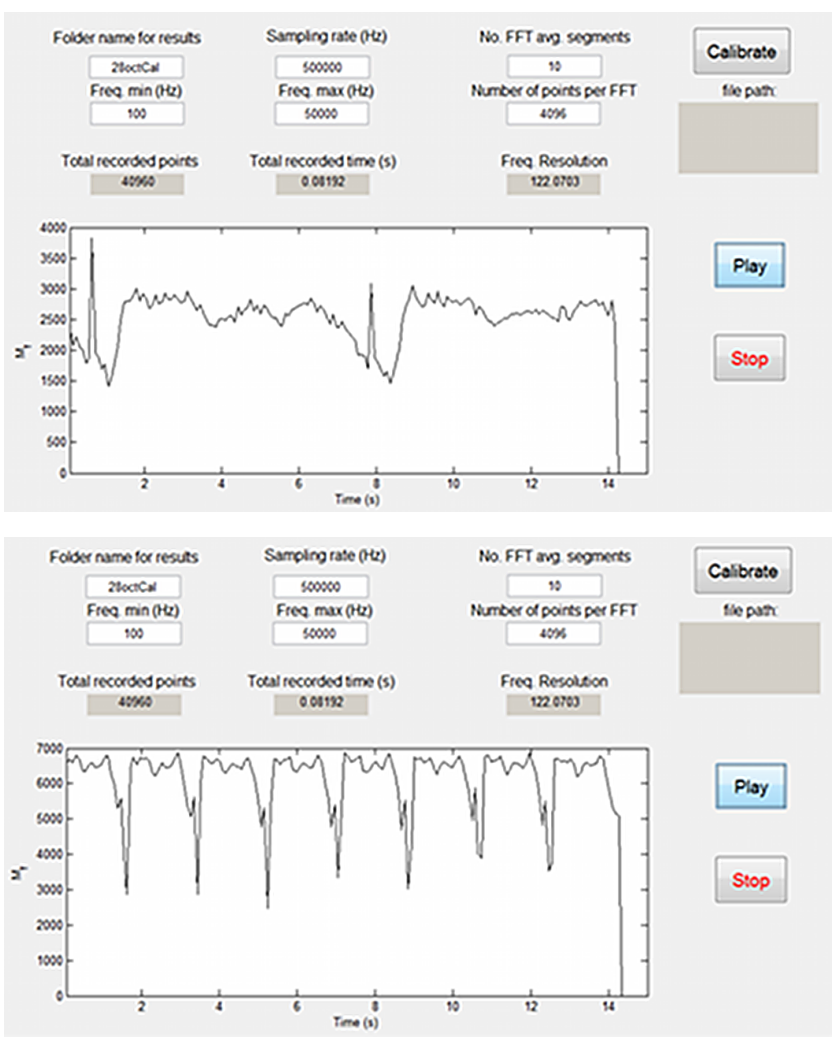

Fig. 7: Front panel of system's software for real-time validation with analog pump's potentiometer at minimal (top) and maximal position (bottom).

\section{CONCLUSION}

We proposed a simple optoelectronic sensor for non-steady flow measurements based on optical feedback interferometry in a commercial semiconductor laser. It was validated in realtime and with post-processing using a straightforward signal processing without analog signal conditioning associated. The OFI sensor demonstrated robust capability to provide information on the flow intermittency in real-time. 


\section{ACKNOWLEDGMENT}

This research was supported by the French Embassy in Havana and COST action BM1205.

\section{REFERENCES}

[1] T. Bosch, C. Bès, L. Scalise, and G. Plantier, "Optical feedback interferometry," in Encyclopedia of Sensors. American Scientific Publishers, 2006, vol. 7, pp. 107 - 126.

[2] C. H. Cheng, L. C. Lin, and F. Y. Lin, "Self-mixing dual-frequency laser doppler velocimeter," Opt. Express, vol. 22, pp. 3600-3610, 2014.

[3] M. Norgia, A. Magnani, and A. Pesatori, "High resolution self-mixing laser rangefinder," Rev. Sci. Instrum., vol. 83, p. 045113, 2012.

[4] U. Zabit, R. Atashkhooei, T. Bosch, S. Royo, F. Bony, and A. D. Rakic, "Adaptive self-mixing vibrometer based on a liquid lens," Opt. Lett., vol. 14, pp. $1278-1280,2010$.

[5] H. Hao, D. Guo, M. Wang, W. Xia, and X. Ni, "Micro-displace sensor based on self-mixing interfrence of the fiber laser with phase modulation," Photonic sensors, vol. 4, pp. 379-384, 2014.

[6] A. L. Arriaga, F. Bony, and T. Bosch, "Speckle-insensitive fringe detection method based on hilbert transform for self-mixing interferometry," Appl. Opt., vol. 53, pp. 6954-6962, 2014.

[7] S. K. Ozdemir, I. Ohno, and S. Shinohara, "A comparative study for the assessment on blood flow measurement using self-mixing laser speckle interferometer," IEEE Trans. Instrum. Meas., vol. 57, pp. 355-363, 2008
[8] L. Krehut, J. Hast, E. Alarousu, and R. Myllyla, "Low cost velocity sensor based on the self-mixing effect in a laser diode," Opto-elect. Rev., vol. 11, pp. 313-319, 2003.

[9] L. Campagnolo, M. Nikolic, J. Perchoux, Y. L. Lim, K. Bertling, K. Loubiere, L. Prat, A. D. Rakic, and T. Bosch, "Flow profile measurement in microchannel using the optical feedback interferometry sensing technique," Microfluid Nanofluid, vol. 14, pp. 113-119, 2013.

[10] R. Kliese, Y. L. Lim, T. Bosch, and A. D. Rakic, "GaN laser self-mixing velocimeter for measuring slow flows," Opt. Lett., vol. 35, pp. 814-816, 2010

[11] A. Quotb, E. E. Ramirez-Miquet, C. Tronche, and J. Perchoux, "Optical feedback interferometry sensor for flow characterization inside ex-vivo vessel," in IEEE Sensors, 2014, pp. 362-365.

[12] M. E. Nikolic, Y. L. Lim, K. Bertling, and A. D. Rakic, "Self-mixing laser Doppler flow sensor: an optofluidic implementation," Appl. Opt., vol. 52, pp. 8128-8133, 2013.

[13] S. Roman, S. Lorthois, P. Duru, and F. Risso, "Velocimetry of red blood cells in microvessels by the dual-slit method: Effect of velocity gradients," Microvasc. Res., vol. 84, pp. 249-261, 2012.

[14] M. Norgia, A. Pesatori, and L. Rovati, "Low-cost optical flowmeter with analog front-end electronics for blood extracorporeal circulators," IEEE Trans. Instrum. Meas., vol. 59, pp. 1233-1239, 2010.

[15] C. Zakian, M. Dickinson, and T. King, "Particle-sizing and flow measurement using self-mixing interferometry with a laser diode," $J$. Opt. A: Pure Appl. Opt., vol. 7, pp. S445-S452, 2005.

[16] M. Nikolic, Y. L. Lim, S. J. Wilson, A. D. Rakic, L. Campagnolo, J. Perchoux, and T. Bosch, "Flow profile measurement in microchannels using changes in laser junction voltage due to self-mixing effect," in Proc. IEEE Sensors Conf., 2011. 